\title{
Características clínicas y de laboratorio en pacientes con cetoacidosis diabética
}

\section{Clinical and laboratory characteristics of patients with diabetic ketoacidosis}

Autores: Marcos A.Vázquez, ${ }^{1}$ Victoria Malvetti Maffei, ${ }^{2}$ Helen López ${ }^{2}$

Resumen: La cetoacidosis diabética (CAD) es una de las complicaciones agudas severas de la diabetes mellitus. La mayoría de los pacientes con CAD tienen diabetes autoinmune tipo 1, sin embargo, los pacientes con diabetes tipo 2 (DM2) también están en riesgo de padecerla durante el estrés catabólico de una enfermedad aguda como traumatismo, cirugía o infección.

Se presenta una serie de 20 casos donde se diagnosticó CAD en sujetos con DM2, la edad media fue 62 años, todas eran mujeres, cuya principal causa precipitante fue la infección. La forma clínica moderada (85\%) fue la predominante.

Palabras claves: diabetes mellitus tipo 2, cetoacidosis diabética

Abstract: The diabetic ketoacidosis (DKA) is one of the severe acute complications of diabetes mellitus. Most patients with DKA have type 1 autoimmune diabetes but patients with type 2 diabetes are also at risk of suffering it during the catabolic stress of an acute disease like a traumatism, surgery or infection. We present a series of twenty cases with DM2 in whom DKA was diagnosed. All were women with a mean age of 62 years, being infection the main cause. The moderate clinical form was predominant (85\%).

Keywords: type 2 diabetes mellitus, diabetic ketoacidosis

\section{Introducción}

La CAD es una entidad asociada a la diabetes mellitus tipo 1. Sin embargo, algunos estudios han demostrado incidencias elevadas en pacientes con DM2, sobre todo en población afroamericana ${ }^{1,2,3,4}$

En nuestro medio se observan casos de CAD en DM2, por lo que es importante conocer las características clínicas de la CAD en pacientes latinoamericanos. Entre los factores precipitantes para el desarrollo de la misma, la infección es la de mayor importancia con un 25 a 56\%, seguida de la falta de administración de insulina en pacientes ya conocidos diabéticos. ${ }^{2,45}$ Otras causas corresponderían a trastornos de personalidady

\footnotetext{
${ }^{1}$ Especialista en Medicina Interna. Servicio de Urgencias del Hospital de Clínicas. F.C.M. - U.N.A.

${ }^{2}$ Especialista en Endocrinología. Primera Cátedra de Clínica Médica. F.C.M. - U.N.A.

Autor correspondiente:

Dr. Marcos A. Vázquez

Dirección: Hospital de Clínicas. San Lorenzo, Paraguay

Teléfono: +(595) 971947590

Correo electrónico : proyectosss2000@yahoo.com.ar
} 
de alimentación, accidentes cerebrovasculares, pancreatitis aguda, isquemia mesentérica entre otras. La mortalidad de la cetoacidosis diabética es consecuencia, por una parte, de la gravedad de las enfermedades que la acompañan y por otra, de los graves trastornos metabólicos que la cetoacidosis produce. La mortalidad oscila en las series más importantes entre 5 y $10 \%{ }^{4,5,6}$

\section{Objetivos}

- Determinar las características clínicas y de laboratorio de los casos de CAD en pacientes con DM2

\section{Material y métodos}

Diseño: observacional corte transversal tipo descriptivo

Población de estudio: pacientes hospitalizados por cetoacidosis diabética en la Primera Cátedra de Clínica Médica del Hospital de Clínicas, durante el período comprendido entre marzo 2008 y marzo 2009.

Criterios de inclusión: Pacientes que cumplan con las criterios diagnósticos de cetoacidosis diabética según la $\mathrm{ADA}^{1}$

Se consideró tres tipos de gravedad según esquema de tabla 1.

\section{TABLA 1}

\section{Gravedad de la cetoacidosis diabética ${ }^{1}$}

\begin{tabular}{lccc}
\hline Cetoacidosis diabética & Leve & Moderada & Severa \\
\hline Glicemia & $>250 \mathrm{mg} / \mathrm{dL}$ & $>250 \mathrm{mg} / \mathrm{dL}$ & $>250 \mathrm{mg} / \mathrm{dL}$ \\
pH arterial & $7,25-7,30$ & $7,0-7,24$ & $<7,0$ \\
Bicarbonato sérico & $15-18$ & $10-15$ & $<10$ \\
Brecha aniónica & $>10$ & $>12$ & $>12$ \\
Cetonas urinarias & positivas & positivas & positivas \\
Cetonas séricas & positivas & positivas & positivas \\
Estado de conciencia & alerta & somnoliento, estupor & coma \\
Osmo laridad & variable & variable & variable \\
\hline Garber AJ et al. Endocr Pract. 2004 Jan-Feb; 10(1): $77-82$. &
\end{tabular}

Criterios de exclusión: pacientes que no contaban con datos de laboratorio relevantes como gasometría y electrolitos.

Variables: edad, sexo, antecedentes de diagnóstico y tipo de diabetes, presencia o ausencia de factores precipitantes, funciones vitales, estado de conciencia, datos de laboratorio: glicemia, cetonuria, gases arteriales, electrolitos séricos.

Muestreo: no probabilístico, de casos consecutivos

Instrumentos: la información fue obtenida con la revisión de la historia clínica de los pacientes. 
Aspectos éticos: se respetó la confidencialidad de los datos. No se requirió consentimiento informado por haberse trabajado con fichas clínicas.

\section{Resultados}

Durante el período de estudio se presentaron 88 casos de crisis hiperglicémicas en pacientes con DM2, de los cuales 20 casos (23\%) correspondieron a CAD. Entre estos casos, la media de edad fue $62,9 \pm 2$ años (tabla 2). Todas eran mujeres.

Se observó que $15 \%$ debutaron con CAD y el factor precipitante en $60 \%$ fue una causa infecciosa. La mortalidad fue $15 \%$ de todo el grupo estudiado.

Los valores metabólicos de ingreso tuvieron una media de $\mathrm{pH} 7,3 \mathrm{mEq} / \mathrm{L}$, bicarbonato $15 \mathrm{mEq} / \mathrm{L}$, osmolaridad $292 \mathrm{mOsm} / \mathrm{L}$, glicemia $336 \mathrm{mg} / \mathrm{dL}$.

\section{TABLA 2}

\section{Distribución etaria de pacientes con cetoacidosis diabética (n 20)}

\begin{tabular}{ccc}
\hline Edad (años) & Frecuencia & Porcentaje \\
\hline $40-49$ & 2 & $10 \%$ \\
$50-59$ & 5 & $25 \%$ \\
$60-69$ & 7 & $35 \%$ \\
$70-79$ & 5 & $25 \%$ \\
80 y más & 1 & $5 \%$ \\
\hline
\end{tabular}

El factor precipitante más frecuente fue la infección (gráfico 1).

\section{GRÁFICO 1}

\section{Factores precipitantes de cetoacidosis diabética (n 20)}

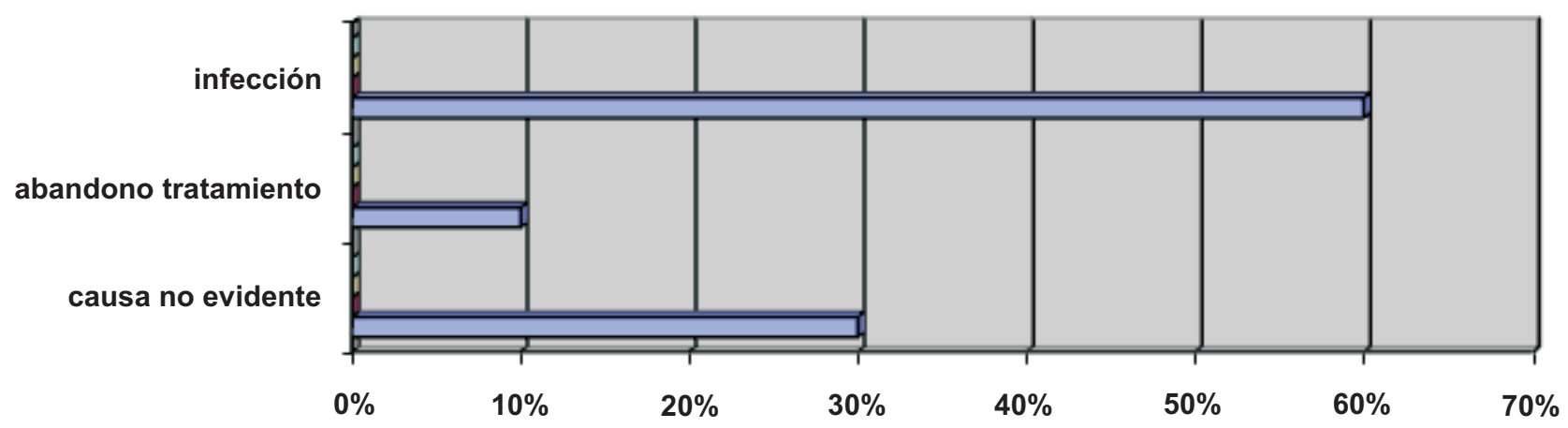

En relación al tipo de infección predominante se encuentra las vias urinarias con 51,2\%.

Entre los datos laboratoriales, llamó la atención la acidosis con bicarbonato bajo (tabla 3) 


\section{TABLA 3}

\section{Datos laboratoriales con cetoacidosis diabética (n 20)}

\begin{tabular}{lc}
\hline \multicolumn{1}{c}{ Parámetros } & Media \pm DS \\
\hline $\mathrm{pH}$ & $7,33 \pm 0,072$ \\
$\mathrm{HCO} 3$ & $15 \pm 4,87$ \\
Glucosa & $336 \pm 96,1$ \\
Osmolaridad & $292 \pm 24,5$ \\
$\mathrm{Na}$ & $135,2 \pm 9,1$ \\
$\mathrm{~K}$ & $4,16 \pm 0,69$ \\
Anion gap & $14,5 \pm 5,7$ \\
\hline
\end{tabular}

Según la forma de gravedad de CAD, se constató 85\% de tipo moderado, 10\% leve y 5\% de tipo severo.

\section{Discusión}

La CAD en DM2 es una condición que se detecta en la Primera Cátedra de Clínica Médica del Hospital de Clínicas. Se presenta en edades avanzadas y el principal factor precipitante es la infección, coincidente con la mayoría de los reportes.

La forma de presentación moderada (85\%), de acuerdo a los criterios de la ADA, es la más frecuente, acorde a otras referencias. ${ }^{2,6}$

\section{Referencias bibliográficas}

1.Garber AJ, Moghissi ES, Bransome ED Jr, Clark NG, Clement S, Cobin RH et al. American College of Endocrinology position statement on inpatient diabetes and metabolic control. Endocr Pract. 2004 Jan-Feb; 10(1): 77-82.

2.American Diabetes Association. Standards of medical care in diabetes 2006. Diabetes Care. 2006 Jan; 29 Suppl 1: S4-42.

3.Adrogué HJ, Madias NE. Management of life-threatening acid-base disorders. First of two parts. N Engl J Med. 1998 Jan $1 ; 338(1): 26-34$.

4.Fowler M. Hyperglycemic Crisis in Adults: Pathophysiology, Presentation, Pitfalls, and Prevention. Clinical Diabetes 2009; 27(1): 19-23

5.Kitabchi AE, Umpierrez GE, Murphy MB, Barrett EJ, Kreisberg RA, Malone JI, Wall BM. Management of hyperglycemic crises in patients with diabetes. Diabetes Care. 2001 Jan; 24(1): 131-53.

6.Magee MF, Bhatt BA. Management of decompensated diabetes. Diabetic ketoacidosis and hyperglycemic hyperosmolar syndrome. Crit Care Clin. 2001 Jan; 17(1): 75-106. 